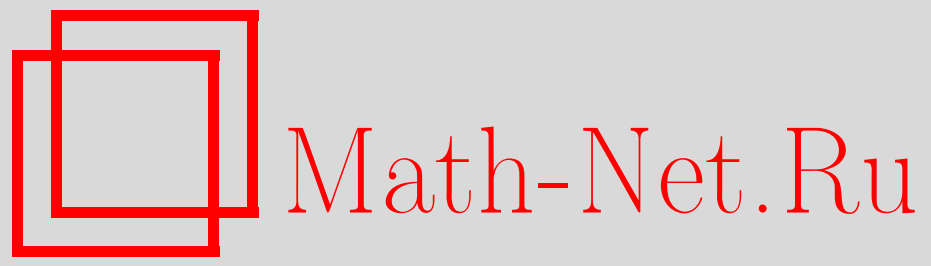

И. А. Дынников, Трехстраничный подход в теории узлов. Кодирование и локальные движения, Функи. анализ и его прил., 1999, том 33, выпуск 4, 25-37

DOI: https://doi.org/10.4213/faa378

Использование Общероссийского математического портала MathNet.Ru подразумевает, что вы прочитали и согласны с пользовательским соглашением

http://www.mathnet.ru/rus/agreement

Параметры загрузки:

IP: 52.23 .180 .231

26 апреля 2023 г., 14:46:35

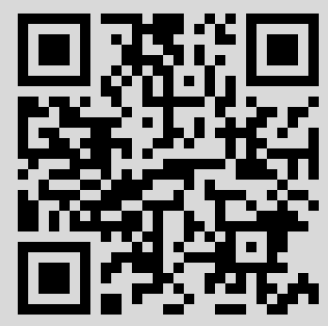


Функциональньй анализ и его приложения

1999, т. 33, вып. 4, с. 25-37

УДК 515.164.63

\title{
Трехстраничный подход в теории узлов. Кодирование и локальные движения*
}

\author{
(c) 1999. И. А. Дынников
}

В настоящей работе предлагается новый комбинаторный подход в теории узлов, основанный на вложении узлов и зацеплений в объединение трех полуплоскостей с общей границей. Сама по себе идея вложения узлов в «книгу» крайне естественна и рассматривалась еще в [1]. Среди недавних работ о вложениях в книгу с неограниченным числом страниц мы упомянем [2] и [3] (см. также ссылки в этих работах).

Ограничение числа страниц тремя (или любым другим числом $\geqslant 3$ ) дает удобный способ кодирования зацеплений словами в конечном алфавите. Для соответствуюших слов мы указываем конечный набор локальных замен, реализующий эквивалентность зацеплений по аналогии с движениями Райдемайстера для плоских диаграмм зацеплений.

\section{§1. Трехстраничные диаграммы}

Пусть $P_{1}, P_{2}, P_{3}$ - три полуплоскости в евклидовом пространстве $\mathbb{R}^{3}$, границы которых совпадают: $\partial P_{1}=\partial P_{2}=\partial P_{3}=l$ (рис. 1 ). Обозначим их объединение $P_{1} \cup P_{2} \cup P_{3}$ через $\mathbb{Y}$. На прямой $l$ выберем ориентацию.

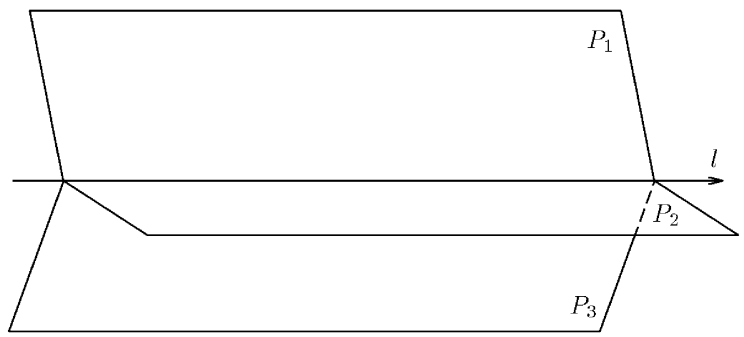

Рис. 1. Трехстраничная книга $\mathbb{Y}=P_{1} \cup P_{2} \cup P_{3}$

В настоящей работе под ломаной линией мы подразумеваем одномерное PL-подмногообразие в $\mathbb{R}^{3}$ (не обязательно связное и, возможно, пустое), каждая компонента которого компактна.

ОПРЕДЕЛЕНИЕ 1. Трехстраничной диаграммой зачепления мы назовем замкнутую ломаную линию $L \subset \mathbb{Y}$, удовлетворяющую следующим двум условиям:

1) трансверсальность к прямой $l$ : пересечение $L \cap l$ конечно, $L \cap l=A_{1} \cup \cdots \cup$ $A_{m}$, и два ребра ломаной $L$, примыкающие к вершине $A_{k}$, лежат в различных полуплоскостях $P_{i}$ для любого $k \leqslant m$;

* Работа выполнена при частичной поддержке Российского фонда фундаментальных исследований (грант № 99-01-00090). 
2) монотонность: ограничение ортогональной проекции $\mathbb{R}^{3} \rightarrow l \cong \mathbb{R}$ на каждую из связных компонент пересечения $L \cap P_{i}$ является монотонной функцией для любого $i=1,2,3$.

Пример трехстраничной диаграммы зацепления показан на рис. 2.

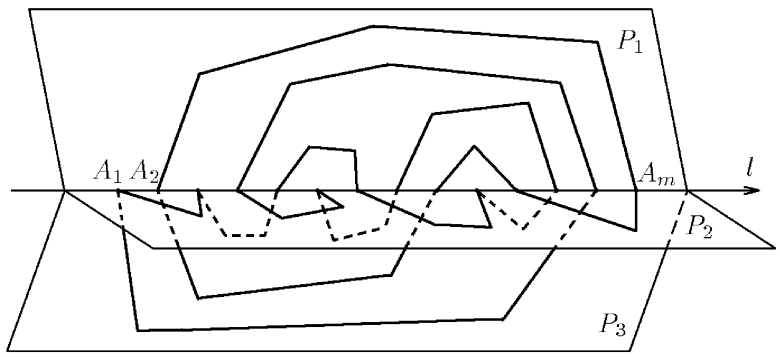

Рис. 2. Трехстраничная диаграмма зацепления

Если ломаная $L$ в определении 1 ориентирована, то мы называем ее ориентированной трехстраничной диаграммой. Число вершин $m=m(L)=\#(L \cap l)$ на прямой $l$ называется сложностью диаграммы $L$. Связные компоненты пересечений $L \cap P_{i}$ называются ребрами диаграммы $L$. Условие 2$)$ в определении 1 означает, что каждое ребро взаимно однозначно проецируется на отрезок, соединяющий ее концевые точки. Отсюда, в частности, следует, что каждая связная компонента трехстраничной диаграммы пересекает прямую $l$, которую мы будем называть линией переплета.

Если ребро соединяет соседние точки $A_{i}$ и $A_{i+1}$ или крайние точки $A_{1}$ и $A_{m}$, то оно называется тривиальным (мы предполагаем, что вершины $A_{i} \in L \cap l$ занумерованы в соответствии с направлением линии переплета $l$ ).

Каждая трехстраничная диаграмма зацепления очевидным образом задает некоторое зацепление в $\mathbb{R}^{3}:$ нужно только взять композицию вложений $L \subset$ $\mathbb{Y} \subset \mathbb{R}^{3}$ и «забыть» про $\mathbb{Y}$. Введем теперь преобразования трехстраничных диаграмм, приводящие к изотопным зацеплениям. Обозначим плоскость $\mathbb{Y}-\left(P_{i}-l\right)$ через $\Pi_{i}, i=1,2,3$. Пусть $L$ - некоторая трехстраничная диаграмма.

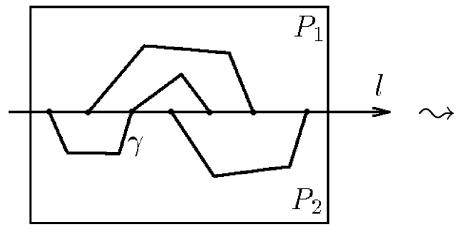

$L$

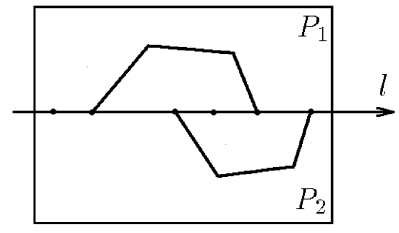

$L-\gamma$

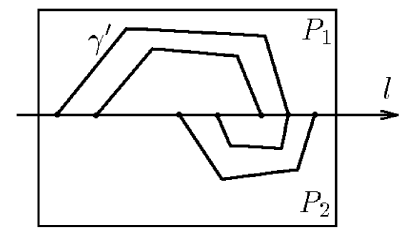

$L^{\prime}$

Рис. 3. Простое движение $(i=3)$

ОПРЕдЕЛЕНИЕ 2. Скажем, что трехстраничная диаграмма $L^{\prime}$ nолучается из $L$ простым движением в плоскости $\Pi_{i}, 1 \leqslant i \leqslant 3$, если для некоторой связной компоненты $\gamma$ пересечения $L \cap \Pi_{i}$ и некоторой связной компоненты $\gamma^{\prime}$ пересечения $L^{\prime} \cap \Pi_{i}$ мы имеем (см. рис. 3 )

$$
L-\gamma=L^{\prime}-\gamma^{\prime}
$$


ТЕОРема 1. Любое зачепление можно представить трехстраничной диаграммой. Две трехстраничные диаграммы представляют изотопные зачепления тогда и только тогда, когда одну можно получить из другой конечныцм иислом простых движений.

Оставшаяся часть параграфа посвящена доказательству этой теоремы. Очевидно, что простые движения сохраняют класс изотопии зацепления. Поэтому нужно только доказать, что простых движений достаточно, чтобы реализовать любую изотопию между двумя трехстраничными диаграммами. Мы сделаем это в три шага.

Сначала мы определим специальный класс трехстраничных диаграмм и покажем, как по плоской диаграмме зацепления построить специальную трехстраничную диаграмму, представляющую то же зацепление (таким образом, мы попутно докажем первое утверждение теоремы). Обращение плоской диаграммы в трехстраничную не единственно, и следующим шагом мы покажем, что различные способы обращения, а также движения Райдемайстера приводят к эквивалентным трехстраничным диаграммам, т.е. диаграммам, получающимся друг из друга конечным числом простых движений. И наконец, мы покажем, что любая трехстраничная диаграмма эквивалентна некоторой специальной трехстраничной диаграмме.

Шаг 1. Скажем, что данная трехстраничная диаграмма зацепления специальнал, если каждое ее ребро в полуплоскости $P_{2}$ имеет длину 2 , т. е. соединяет некоторую вершину $A_{i}$ с вершиной $A_{i+2}$.

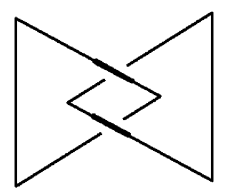

(a)

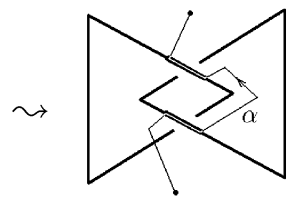

(b)

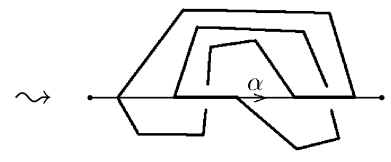

(c)

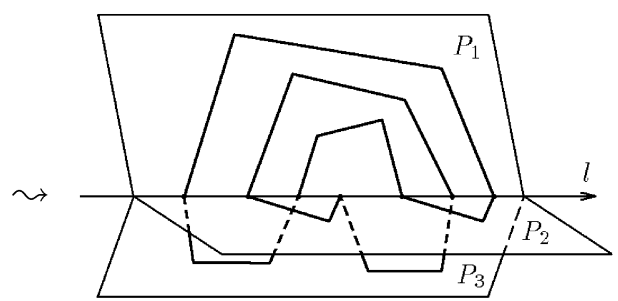

(d)

Рис. 4. Превращение плоской диаграммы зацепления в трехстраничную диаграмму

Пусть $D$ - плоская диаграмма зацепления. Для каждого перекрестка диаграммы $D$ отметим маленький отрезок верхней дуги («мост») (см. рис. 4(a)). Пусть $\alpha$ - произвольный ориентированный путь в плоскости диаграммы $D$, обладающий следующими свойствами: 
1) $\alpha$ проходит по каждому мосту ровно один раз;

2) $\alpha$ пересекает оставшуюся часть диаграммы $D$ трансверсально;

3) $\alpha$ не имеет самопересечений;

4) начало и конец пути $\alpha$ лежат вне $D$ (рис. 4(b)).

Диаграмму $D$, снабженную такой кривой $\alpha$, будем называть линеаризованной диаграммой зацепления.

Между изотопическими классами линеаризованных плоских диаграмм и изотопическими классами трехстраничных диаграмм имеется очевидное взаимно однозначное соответствие. Для превращения линеаризованной плоской диаграммы в трехстраничную нужно деформировать плоскость так, чтобы путь $\alpha$ оказался отрезком некоторой прямой, затем приклеить вдоль этой прямой полуплоскость, и, наконец, вытолкнуть мосты в приклеенную полуплоскость (рис. 4(c)-(d)).

Шаг 2. Доказательство следующего утверждения очевидно.

Лемма 1. Две линеаризованнье плоские диаграммы $(D, \alpha)$ и $\left(D^{\prime}, \alpha^{\prime}\right)$ представляют изотопные зачепления тогда и только тогда, когда одну можно получить из другой конечной последовательностью преобразований следующих типов:

mun A

A1:

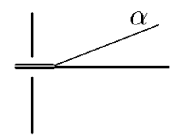

A2:

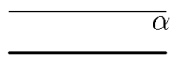

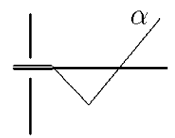

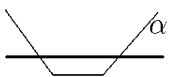

mun $\mathrm{B}$
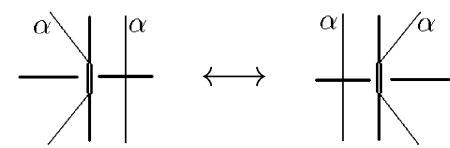

mип C (движения Райдемайстера)

C1:
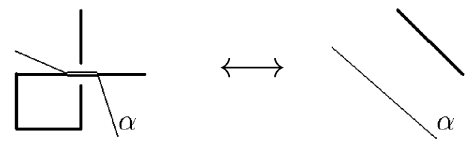

C2:
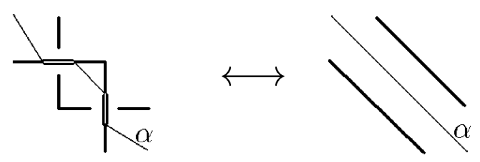

C3:
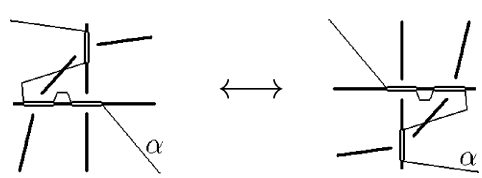

Лемма 2. Преобразования специальных трехстраничных диаграмм, соответствуюшие перечисленным в лемме 1 преобразованиям линеаризованных диаграмм, представляются в виде композичии простьх движений. 
ДокАЗАТЕЛЬСТво. Легко видеть, что каждое из преобразований типа А или В преврашается в одно простое движение соответствующих трехстраничных диаграмм. Преобразования типа $\mathrm{C} 1$ и $\mathrm{C} 2$ разлагаются в композицию соответственно двух и трех простых движений, которые также очевидны.

Мы укажем последовательность простых движений, дающих преобразование типа С3, единственный нетривиальный случай. Для определенности предположим, что путь $\alpha$ на рисунке в лемме 1 направлен из левого верхнего в правый нижний угол. Первые четыре диаграммы, т.е. первые три простых движения, изображены на рис. 5. Пятая, шестая, седьмая и восьмая диаграммы симметричны четвертой, третьей, второй и первой диаграммам соответственно относительно прямой, лежащей в плоскости страницы $P_{2}$ и перпендикулярной линии переплета $l$. Первое преобразование есть простое движение в плоскости $\Pi_{1}$, второе - в плоскости $\Pi_{2}$, третье - в плоскости $\Pi_{3}$.
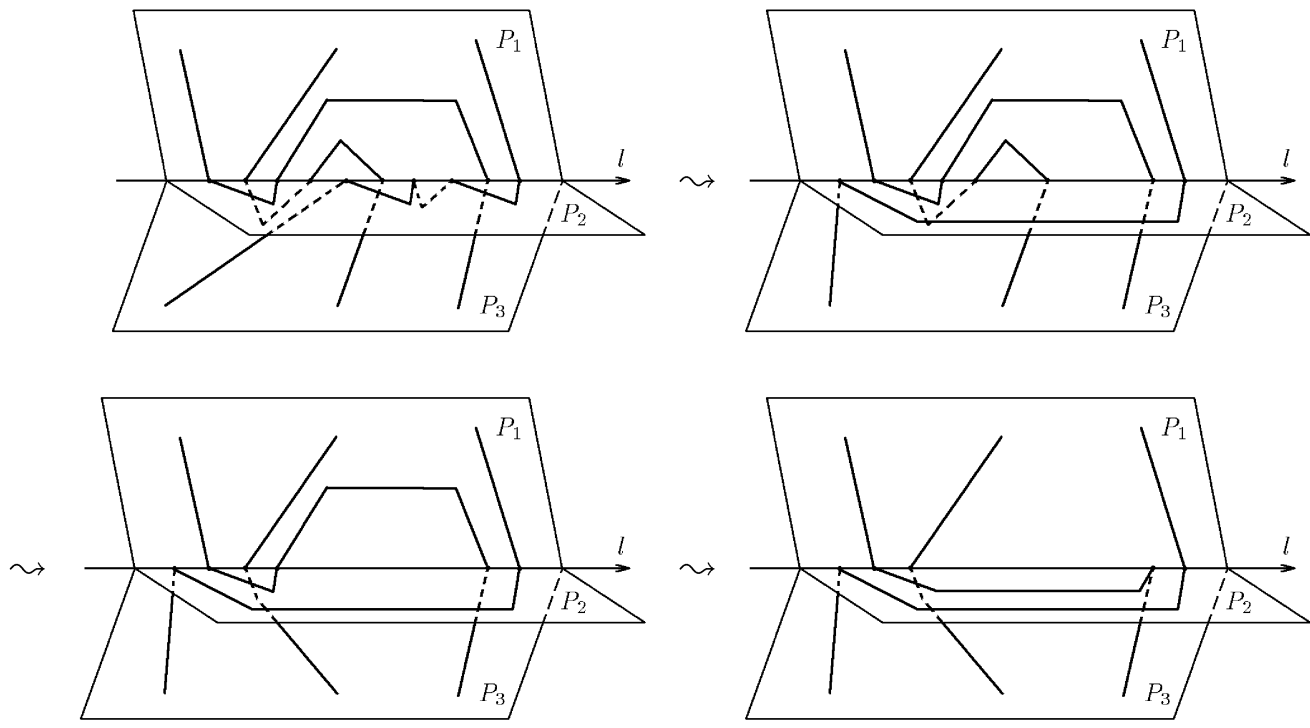

Рис. 5. Простые движения, реализующие движение Райдемайстера типа III

Шаг 3. Для завершения доказательства теоремы 1 осталось установить следующее.

ЛЕмма 3. Любая трехстраничная диаграмма эквивалентна некоторой спеииальной трехстраничной диаграмме.

ДокаЗАТЕЛЬСтво. Очевидно, что, используя простые движения, можно избавиться от всех тривиальных ребер в полуплоскости $P_{2}$. Затем мы будем применять простые движения, показанные на рис. 6 , до тех пор, пока это возможно. Нетрудно видеть, что этот процесс закончится за конечное число шагов, а результат будет специальной трехстраничной диаграммой. 

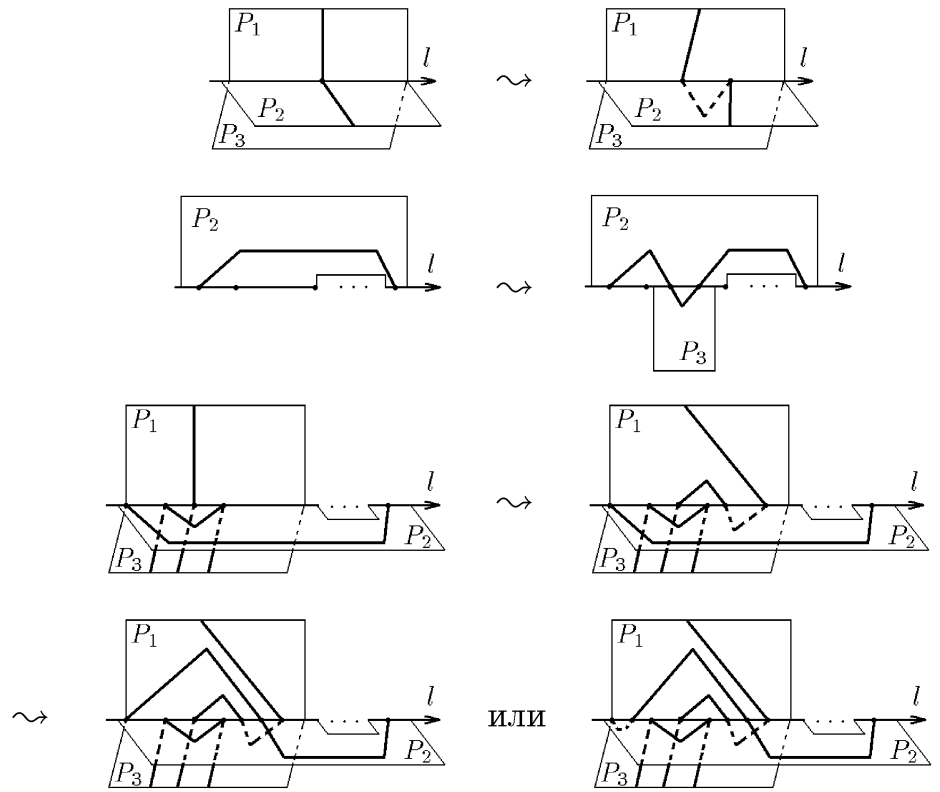

Рис. 6. Приведение трехстраничной диаграммы к специальному виду

\section{§2. Кодировка трехстраничных диаграмм}

Рассмотрим множество $\mathbb{A}$, состоящее из следующих двенадцати векторов:

$$
\begin{aligned}
& a_{1}=\left(\begin{array}{l}
0 \\
1 \\
1
\end{array}\right), \quad b_{1}=\left(\begin{array}{r}
0 \\
1 \\
-1
\end{array}\right), \quad c_{1}=\left(\begin{array}{r}
0 \\
-1 \\
-1
\end{array}\right), \quad d_{1}=\left(\begin{array}{r}
0 \\
-1 \\
1
\end{array}\right), \quad a_{2}=\left(\begin{array}{r}
1 \\
0 \\
1
\end{array}\right), \quad b_{2}=\left(\begin{array}{r}
-1 \\
0 \\
1
\end{array}\right), \\
& c_{2}=\left(\begin{array}{r}
-1 \\
0 \\
-1
\end{array}\right), \quad d_{2}=\left(\begin{array}{r}
1 \\
0 \\
-1
\end{array}\right), \quad a_{3}=\left(\begin{array}{l}
1 \\
1 \\
0
\end{array}\right), \quad b_{3}=\left(\begin{array}{r}
1 \\
-1 \\
0
\end{array}\right), \quad c_{3}=\left(\begin{array}{r}
-1 \\
-1 \\
0
\end{array}\right), \quad d_{3}=\left(\begin{array}{r}
-1 \\
1 \\
0
\end{array}\right) .
\end{aligned}
$$

Пусть дана некоторая неориентированная трехстраничная диаграмма $L$. Обозначим, как и раньше, вершины из $L \cap l$ через $A_{1}, \ldots, A_{m}$, занумеровав их в порядке следования на линии переплета $l$. Каждой вершине $A_{k}$ сопоставим ее mип - вектор $x_{k} \in \mathbb{A}$, определенный по следующему правилу: $i$-я координата вектора $x_{k}$ для $i \in\{1,2,3\}$ равна

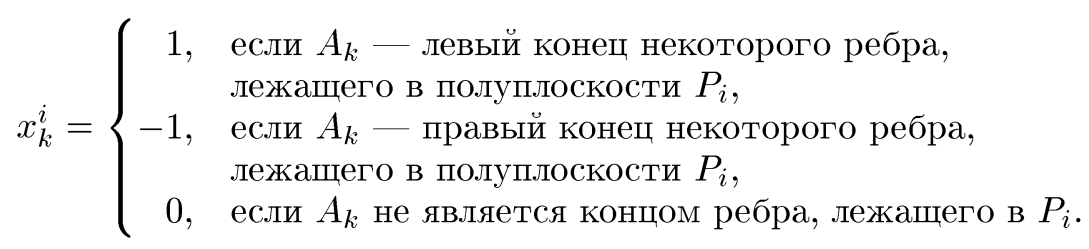

Трехстраничной диаграмме $L$ мы сопоставляем следующее слово в алфавите $\mathbb{A}$ :

$$
w_{L}=x_{1} \ldots x_{m} .
$$

Например, трехстраничной диаграмме зацепления Уайтхеда, показанной на рис. 2, соответствует слово

$$
a_{1} a_{2} d_{1} a_{3} d_{2} d_{1} d_{3} d_{2} d_{2} d_{1} d_{3} c_{2} c_{2} c_{3} .
$$


Обозначим через $W$ множество всех слов в алфавите $\mathbb{A}$, включая пустое слово. Нам понадобится следующее

ОПРЕДЕЛЕНИЕ 3. Для $i \in\{1,2,3\}$ слово $w=x_{1} \ldots x_{m} \in W$ называется $i$-сбалансированньл, если $\sum_{k=1}^{m} x_{k}^{i}=0$ и

$$
\sum_{k=1}^{p} x_{k}^{i} \geqslant 0 \quad \text { для всех } 1 \leqslant p \leqslant k .
$$

Если слово $i$-сбалансированно для всех $i \in\{1,2,3\}$, то мы говорим, что оно полностью сбалансированно.

По-другому $i$-сбалансированность можно определить так: если в последовательности $x_{1}^{i} \ldots x_{m}^{i}$ заменить все «1» на открывающую скобку, а все «-1»на закрывающую, то получится выражение со сбалансированными скобками. Следующее утверждение очевидно.

ПреДлОЖЕНИЕ 1. Слово $w \in W$ имеет вид $w=w_{L}$, аде $L$ - некоторая трехстраничная диаграмма, тогда и только тогда, когда оно полностью сбалансированно. Две неориентированные трехстраничнье диаграммь $L u L^{\prime}$ объемлемо изотопны в $\mathbb{Y}$ тогда и только тогда, когда $w_{L}=w_{L^{\prime}}$.

Таким образом, подмножество $W_{0} \subset W$ полностью сбалансированных слов в алфавите $\mathbb{A}$ классифицирует трехстраничные диаграммы с точностью до объемлемой изотопии в $\mathbb{Y}$. В следующем параграфе мы укажем набор локальных преобразований сбалансированных слов, порождающий отношение эквивалентности трехстраничных диаграмм.

\section{§3. Локальные движения.}

Простые движения, введенные выше, нелокальны. В этом параграфе мы сведем их к«более простым». Для начала мы разложим простые движения в композицию элементарных движений трех типов.

ПРЕДЛОЖЕНИЕ 2. Каждое простое движение реализуется конечной последовательностью простых движений следуюших трех типов:

mun I (раздутие/стяаивание тривиального ребра):

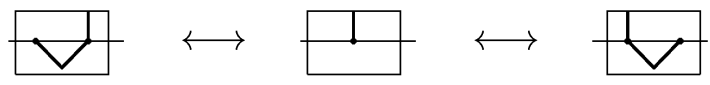

тип II (вставка/удаление тривиального ребра):

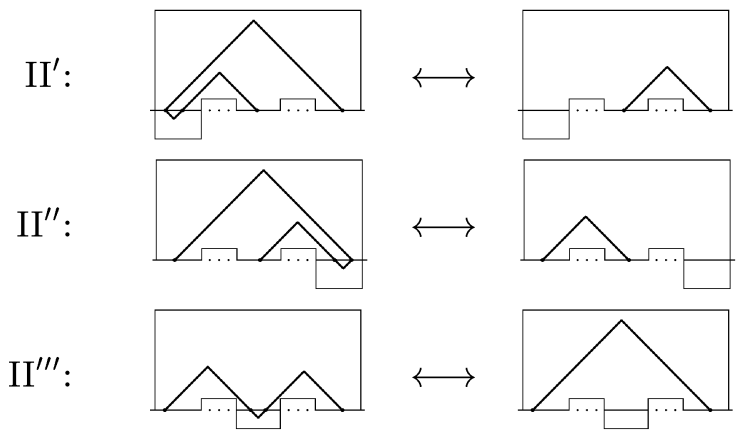


тип III (перестановка параллельных незачепленных ребер):
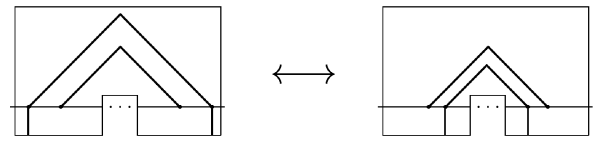

На рисунках выше подразумевается, что хвост, перпендикулярный линии переплета: $\square$, может быть направлен как вправо: $\square$, так и влево: $\triangle$. Если на рисунке показан только один хвост, прикрепленный к данной вершине на линии переплета, то подразумевается, что второй лежит в полуплоскости, не показанной на рисунке.

ДокАЗАТЕльство. Для определенности предположим, что мы хотим выполнить простое движение в плоскости $\Pi_{3}=P_{1} \cup P_{2}$, т. е. заменить некоторую связную компоненту $\gamma$ пересечения $L \cap \Pi_{3}$ на другой путь $\gamma^{\prime} \subset \Pi_{3}, \partial \gamma=\partial \gamma^{\prime}$. Если пути $\gamma$ и $\gamma^{\prime}$ изотопны в $\Pi_{3}-(L-\gamma)$ (например, если $\gamma$ - единственная компонента пересечения $L \cap \Pi_{3}$ ), то проблемы нет: нужно применить конечное число элементарных движений типов I и II.

Препятствием к изотопии (сохраняющей конщевые точки) между $\gamma$ и $\gamma^{\prime}$ могут быть только другие связные компоненты $\gamma_{1}, \ldots, \gamma_{k}$ пересечения $L \cap \Pi_{3}$, отличные от $\gamma$. Чтобы получить из $\gamma$ путь $\gamma^{\prime}$, мы должны осуществлять изотопии в $\Pi_{3}-\left(\bigcup_{i} \gamma_{i}\right)$ и, возможно, перебросить $\gamma$ через $\gamma_{i}$ конечное число раз.

Движение типа III позволяет перебросить $\gamma$ через компоненту $\gamma_{i}$ в случае, когда последняя состоит ровно из одного ребра. Таким образом, для завершения доказательства осталось свести все к случаю, когда каждая компонента $\gamma_{i}$ состоит ровно из одного ребра. Для этого мы каждую вершину $A_{k} \in \gamma_{i}$, находящуюся на стыке двух ребер, одно из которых лежит в $P_{1}$, а другое - в $P_{2}$, раздуем в ребро, лежащее в полуплоскости $P_{3}$.

Заметим, что каждой трехстраничной диаграмме $L$ мы можем сопоставить граф с носителем $L \cup l \cup\{\infty\}$, где точка $\infty$ добавлена, чтобы превратить прямую $l$ в замкнутую окружность. Ребра этого графа естественно раскрасить в четыре цвета: для $i=1,2,3$ ребра в полуплоскости $P_{i}$ раскрашиваются в $i$-й цвет, а вся линия переплета $l$ окрашивается в цвет 4. Такой граф с раскраской, отмеченной точкой $\infty$ и ориентацией на одном из ребер цвета 4 удовлетворяет определенным условиям и содержит всю комбинаторную информацию о трехстраничной диаграмме.

С точки зрения теории таких графов элементарные движения, рассмотренные выше, являются локальными и, по-видимому, могут быть использованы для нахождения инвариантов зацеплений по аналогии с работой [6]. А именно, на каждую вершину графа можно посадить тензор с четырьмя индексами (зависящий от цветов примыкающих ребер), которые соответствуют ребрам, и затем произвести суммирование по всем ребрам. Элементарные движения приводят к алгебраическим соотношениям, которым должен удовлетворять такой тензор, чтобы указанная процедура приводила к изотопическому инварианту. Мы не выписываем здесь этих соотношений, поскольку их алгебраическая природа неясна и ни одного нетривиального решения автору не известно.

Теперь мы предъявим некоторый набор движений, являюшихся локальными для слов в алфавите $\mathbb{A}$. Для удобства мы будем считать индекс у $a_{i}, b_{i}, c_{i}, d_{i}$, $P_{i}$ вычетом по модулю 3. 
Теорема 2. Два сбалансированных слова $w, w^{\prime} \in W_{0}$ представляют изотопные неориентированные зачепления в $\mathbb{R}^{3}$ тогда и только тогда, когда $w^{\prime}$ можно получить из $w$ конечной последовательностью следующих локальных замен подслов:

$$
\begin{aligned}
& \left.\begin{array}{rl}
a_{i+1} d_{i-1} & \leftrightarrow a_{i} \leftrightarrow a_{i-1} b_{i+1}, \\
d_{i+1} d_{i-1} & \leftrightarrow b_{i} \leftrightarrow a_{i-1} c_{i+1}, \\
d_{i+1} c_{i-1} & \leftrightarrow c_{i} \leftrightarrow b_{i-1} c_{i+1}, \\
a_{i+1} c_{i-1} & \leftrightarrow d_{i} \leftrightarrow b_{i-1} b_{i+1},
\end{array}\right\} \\
& a_{i-1} b_{i-1} w_{i} \leftrightarrow w_{i} a_{i-1} b_{i-1}, \\
& d_{i-1} c_{i-1} w_{i} \leftrightarrow w_{i} d_{i-1} c_{i-1}, \\
& \left.d_{i-1} b_{i-1} w_{i} \leftrightarrow w_{i} d_{i-1} b_{i-1},\right\} \\
& \left(b_{i+1} d_{i-1} d_{i+1} b_{i-1}\right) w_{i} \leftrightarrow w_{i}\left(b_{i+1} d_{i-1} d_{i+1} b_{i-1}\right),
\end{aligned}
$$

где $i \in \mathbb{Z}_{3}, \quad w_{i} \in X_{i} u X_{i}=\left\{a_{i}, b_{i}, c_{i}, d_{i}, b_{i-1} a_{i} d_{i-1}, b_{i-1} b_{i} d_{i-1}, b_{i-1} c_{i} d_{i-1}\right.$, $\left.b_{i-1} d_{i} d_{i-1}\right\}$. Все указанные преобразования сохраняют подмножество $W_{0}$ полностью сбалансированных слов.

Для двух слов $u, v \in W$ мы пишем $u \sim v$, если $u$ можно получить из $v$ конечным числом локальных преобразований (1)-(3).

Оставшаяся часть параграфа посвящена доказательству теоремы 2 . Прежде всего заметим, что замены (1) соответствуют раздутию/стягиванию тривиального ребра, а все замены в (2) и (3) имеют вид: $i$-сбалансированное подслово переставляется с подсловом, которое одновременно $(i+1)$-сбалансированно и $(i-1)$-сбалансированно. Легко видеть, что указанные преобразования сохраняют сбалансированность слова и изотопический класс соответствующего зацепления.

Теперь нам нужно доказать, что элементарные движения из предложения 2 сохраняют класс эквивалентности слов по модулю преобразований (1)-(3). Для начала мы выполним некоторые предварительные вычисления.

Лемма 4. Для $i \in \mathbb{Z}_{3}$ мй имеем

$$
\begin{aligned}
& a_{i} d_{i} b_{i} \sim a_{i} \sim a_{i} b_{i} d_{i}, \quad b_{i} d_{i} b_{i} \sim b_{i} \sim a_{i} b_{i} c_{i}, \\
& b_{i} d_{i} c_{i} \sim c_{i} \sim d_{i} b_{i} c_{i}, \quad a_{i} d_{i} c_{i} \sim d_{i} \sim d_{i} b_{i} d_{i}, \\
& a_{i} b_{i} \sim a_{i-1} d_{i-1}, \quad d_{i} c_{i} \sim b_{i-1} c_{i-1}, \quad d_{i} b_{i} \sim b_{i-1} d_{i-1} .
\end{aligned}
$$

ДоказАТЕЛЬСтво. Действительно,

$$
\begin{gathered}
a_{i} d_{i} b_{i} \sim a_{i} b_{i-1} b_{i+1} b_{i} \sim a_{i+1} d_{i-1} \sim a_{i} \sim a_{i-1} b_{i+1} \sim a_{i} d_{i+1} d_{i-1} d_{i} \sim a_{i} b_{i} d_{i}, \\
b_{i} d_{i} b_{i} \sim b_{i} b_{i-1} b_{i+1} b_{i} \sim d_{i+1} d_{i-1} \sim b_{i} \sim a_{i-1} c_{i+1} \sim a_{i} d_{i+1} d_{i-1} c_{i} \sim a_{i} b_{i} c_{i}, \\
b_{i} d_{i} c_{i} \sim b_{i} b_{i-1} b_{i+1} c_{i} \sim d_{i+1} c_{i-1} \sim c_{i} \sim b_{i-1} c_{i+1} \sim d_{i} d_{i+1} d_{i-1} c_{i} \sim d_{i} b_{i} c_{i}, \\
a_{i} d_{i} c_{i} \sim a_{i} b_{i-1} b_{i+1} c_{i} \sim a_{i+1} c_{i-1} \sim d_{i} \sim b_{i-1} b_{i+1} \sim d_{i} d_{i+1} d_{i-1} d_{i} \sim d_{i} b_{i} d_{i}, \\
a_{i} b_{i} \sim a_{i-1} b_{i+1} b_{i} \sim a_{i-1} d_{i-1}, \quad d_{i} c_{i} \sim b_{i-1} b_{i+1} c_{i} \sim b_{i-1} c_{i-1}, \\
d_{i} b_{i} \sim b_{i-1} b_{i+1} b_{i} \sim b_{i-1} d_{i-1} .
\end{gathered}
$$

Лемма 5. Для любых $i \in \mathbb{Z}_{3}, x \in\left\{a_{i}, b_{i}, c_{i}, d_{i}\right\}$ мьи имеем

$$
d_{i-1} b_{i+1} b_{i-1} d_{i+1} x \sim x d_{i-1} b_{i+1} b_{i-1} d_{i+1} .
$$


ДокАЗАТЕЛьство. Действительно,

$d_{i-1} b_{i+1} b_{i-1} d_{i+1} x$

$\sim d_{i-1} b_{i+1} b_{i-1} d_{i+1} b_{i+1} d_{i+1} x \sim d_{i-1} b_{i+1} b_{i-1} d_{i+1} x b_{i+1} d_{i+1}$

$\sim d_{i-1} b_{i+1} b_{i-1} d_{i-1} b_{i-1} d_{i+1} x b_{i+1} d_{i+1} \sim d_{i-1} b_{i+1} b_{i-1} d_{i-1} b_{i-1} b_{i} b_{i-1} x d_{i-1} d_{i} d_{i+1}$

$\sim d_{i-1} b_{i+1} b_{i-1} b_{i} b_{i-1} x d_{i-1} d_{i} d_{i-1} b_{i-1} d_{i+1} \sim d_{i-1} b_{i+1} b_{i-1} d_{i+1} x b_{i+1} d_{i-1} b_{i-1} d_{i+1}$

$\sim d_{i-1} b_{i+1} b_{i-1} d_{i+1} x b_{i+1} d_{i+1} b_{i+1} d_{i-1} b_{i-1} d_{i+1}$

$\sim d_{i-1} b_{i+1} b_{i-1} d_{i+1} x b_{i+1} d_{i-1} d_{i+1} b_{i+1} b_{i-1} d_{i+1}$

$\sim d_{i-1} b_{i+1} b_{i-1} d_{i+1} x b_{i+1} b_{i+1} b_{i} d_{i+1} b_{i+1} b_{i-1} d_{i+1}$

$\sim d_{i-1} b_{i+1} b_{i-1} d_{i+1} x b_{i+1} d_{i-1} d_{i} b_{i} d_{i+1} b_{i+1} b_{i-1} d_{i+1}$

$\sim d_{i-1} b_{i+1} b_{i-1} d_{i+1} x b_{i+1} d_{i-1} d_{i+1} d_{i} b_{i} b_{i+1} b_{i-1} d_{i+1}$

$\sim d_{i-1} b_{i+1} b_{i-1} d_{i+1} x b_{i+1} d_{i-1} d_{i+1} b_{i-1} d_{i-1} b_{i+1} b_{i-1} d_{i+1}$

$\sim d_{i-1} b_{i+1} b_{i-1} d_{i+1} b_{i+1} d_{i-1} d_{i+1} b_{i-1} x d_{i-1} b_{i+1} b_{i-1} d_{i+1}$

$\sim d_{i-1} b_{i+1} d_{i+1} b_{i+1} b_{i-1} d_{i-1} d_{i+1} b_{i-1} x d_{i-1} b_{i+1} b_{i-1} d_{i+1}$

$\sim d_{i-1} b_{i+1} b_{i-1} d_{i-1} d_{i+1} b_{i-1} x d_{i-1} b_{i+1} b_{i-1} d_{i+1}$

$\sim d_{i-1} b_{i+1} d_{i} b_{i} d_{i+1} b_{i-1} x d_{i-1} b_{i+1} b_{i-1} d_{i+1}$

$\sim d_{i-1} b_{i+1} d_{i+1} d_{i} b_{i} b_{i-1} x d_{i-1} b_{i+1} b_{i-1} d_{i+1}$

$\sim d_{i-1} b_{i+1} d_{i+1} d_{i} d_{i+1} x d_{i-1} b_{i+1} b_{i-1} d_{i+1} \sim d_{i-1} b_{i+1} d_{i+1} b_{i-1} x d_{i-1} b_{i+1} b_{i-1} d_{i+1}$

$\sim d_{i-1} d_{i-1} b_{i-1} b_{i-1} x d_{i-1} b_{i+1} b_{i-1} d_{i+1} \sim d_{i-1} b_{i-1} x d_{i-1} d_{i-1} b_{i-1} b_{i+1} b_{i-1} d_{i+1}$

$\sim d_{i-1} b_{i-1} x d_{i-1} d_{i-1} d_{i} b_{i-1} d_{i+1} \sim d_{i-1} b_{i-1} x d_{i-1} b_{i+1} b_{i-1} d_{i+1}$

$\sim x d_{i-1} b_{i-1} d_{i-1} b_{i+1} b_{i-1} d_{i+1} \sim x d_{i-1} b_{i+1} b_{i-1} d_{i+1}$.

ЛЕмма 6. Для $i \in \mathbb{Z}_{3}$ мьи имеем

$$
\begin{aligned}
b_{i-1}^{2} a_{i} d_{i-1}^{2} & \sim b_{i-1} a_{i} d_{i-1} d_{i}^{2} b_{i-1} b_{i} d_{i-1} b_{i}, \\
b_{i-1}^{2} b_{i} d_{i-1}^{2} & \sim b_{i-1} b_{i} d_{i-1} d_{i}^{2} b_{i-1} b_{i} d_{i-1} b_{i} \\
b_{i-1}^{2} c_{i} d_{i-1}^{2} & \sim d_{i} b_{i-1} d_{i} d_{i-1} b_{i}^{2} b_{i-1} c_{i} d_{i-1}, \\
b_{i-1}^{2} d_{i} d_{i-1}^{2} & \sim d_{i} b_{i-1} d_{i} d_{i-1} b_{i}^{2} b_{i-1} d_{i} d_{i-1} .
\end{aligned}
$$

\section{ДокАЗАТЕЛьство. Действительно,}

$b_{i-1}^{2} a_{i} d_{i-1}^{2} \sim b_{i-1}^{2} a_{i} d_{i} b_{i} d_{i-1}^{2} \sim b_{i-1}^{2} a_{i+1} b_{i+1} b_{i} d_{i-1}^{2} \sim b_{i-1} a_{i+1} b_{i+1} b_{i-1} b_{i} d_{i-1}^{2}$

$\sim b_{i-1} a_{i} d_{i} b_{i-1} b_{i} d_{i-1}^{2} \sim b_{i-1} a_{i} d_{i} b_{i-1} b_{i} d_{i-1} b_{i+1} b_{i}$

$\sim b_{i-1} a_{i} b_{i+1} d_{i} b_{i-1} b_{i} d_{i-1} b_{i} \sim b_{i-1} a_{i} d_{i-1} d_{i}^{2} b_{i-1} b_{i} d_{i-1} b_{i}$,

$b_{i-1}^{2} b_{i} d_{i-1}^{2} \sim b_{i-1}^{2} b_{i} d_{i} b_{i} d_{i-1}^{2} \sim b_{i-1}^{2} d_{i+1} b_{i+1} b_{i} d_{i-1}^{2} \sim b_{i-1} d_{i+1} b_{i+1} b_{i-1} b_{i} d_{i-1}^{2}$

$\sim b_{i-1} b_{i} d_{i} b_{i-1} b_{i} d_{i-1}^{2} \sim b_{i-1} b_{i} d_{i} b_{i-1} b_{i} d_{i-1} b_{i+1} b_{i}$

$\sim b_{i-1} b_{i} b_{i+1} d_{i} b_{i-1} b_{i} d_{i-1} b_{i} \sim b_{i-1} b_{i} d_{i-1} d_{i}^{2} b_{i-1} b_{i} d_{i-1} b_{i}$,

$b_{i-1}^{2} c_{i} d_{i-1}^{2} \sim b_{i-1}^{2} d_{i} b_{i} c_{i} d_{i-1}^{2} \sim b_{i-1}^{2} d_{i} d_{i+1} c_{i+1} d_{i-1}^{2} \sim b_{i-1}^{2} d_{i} d_{i-1} d_{i+1} c_{i+1} d_{i-1}$

$\sim b_{i-1}^{2} d_{i} d_{i-1} b_{i} c_{i} d_{i-1} \sim d_{i} d_{i+1} b_{i-1} d_{i} d_{i-1} b_{i} c_{i} d_{i-1}$

$\sim d_{i} b_{i-1} d_{i} d_{i-1} b_{i} d_{i+1} c_{i} d_{i-1} \sim d_{i} b_{i-1} d_{i} d_{i-1} b_{i}^{2} b_{i-1} c_{i} d_{i-1}$,

$b_{i-1}^{2} d_{i} d_{i-1}^{2} \sim b_{i-1}^{2} d_{i} b_{i} d_{i} d_{i-1}^{2} \sim b_{i-1}^{2} d_{i} d_{i+1} b_{i+1} d_{i-1}^{2} \sim b_{i-1}^{2} d_{i} d_{i-1} d_{i+1} b_{i+1} d_{i-1}$

$\sim b_{i-1}^{2} d_{i} d_{i-1} b_{i} d_{i} d_{i-1} \sim d_{i} d_{i+1} b_{i-1} d_{i} d_{i-1} b_{i} d_{i} d_{i-1}$

$\sim d_{i} b_{i-1} d_{i} d_{i-1} b_{i} d_{i+1} d_{i} d_{i-1} \sim d_{i} b_{i-1} d_{i} d_{i-1} b_{i}^{2} b_{i-1} d_{i} d_{i-1}$. 
Следующее утверждение, обобщающее лемму 3, играет ключевую роль в доказательстве теоремы 2.

Лемма 7. Для любого $i$-сбалансированного слова $w \in W$ существует набор $w_{1}, \ldots, w_{k}$ слов из $X_{i}$, такой, что $w \sim w_{1} \ldots w_{k}$.

Обратное утверждение очевидно: если $w_{j} \in X_{i}, j=1, \ldots, k$, то слово $w_{1} \ldots w_{k}$ является $i$-сбалансированным. Мы будем обозначать множество всех $i$-сбалансированных слов через $\bar{X}_{i}$.

ДокАЗАТЕЛЬСТво ЛЕммы 7. Пусть $w \in W$ - некоторое $i$-сбалансированное слово. Произведем в нем всевозможные замены (1) из следующего списка

$$
\begin{aligned}
& a_{i-1} \leadsto a_{i} d_{i+1}, \quad a_{i+1} \leadsto a_{i} b_{i-1}, \quad c_{i-1} \leadsto b_{i+1} c_{i}, \\
& c_{i+1} \leadsto d_{i-1} c_{i}, \quad b_{i+1} \leadsto d_{i-1} d_{i}, \quad d_{i+1} \leadsto b_{i} b_{i-1} \text {. }
\end{aligned}
$$

Мы получим новое слово $w^{\prime}$, содержащее только буквы $a_{i}, b_{i}, c_{i}, d_{i}, b_{i-1}, d_{i-1}$. Кроме того, поскольку слово $w$ было сбалансированным, новое слово $w^{\prime}$ обладает следующим свойством: если заменить в нем все вхождения буквы $b_{i-1}$ на открывающую скобку (, а все вхождения $d_{i-1}$ - на закрывающую скобку ), то получится выражение со сбалансированными скобками.

Теперь, чтобы преобразовать слово $w^{\prime}$ в композицию слов из $X_{i}$, будем применять следующие локальные замены до тех пор, пока возможно:

$$
\begin{gathered}
b_{i-1} d_{i-1} \leadsto d_{i} b_{i}, \\
b_{i-1} u v \leadsto b_{i-1} d_{i-1} b_{i-1} u v \leadsto b_{i-1} u d_{i-1} b_{i-1} v, \quad \text { где } u, v \in X_{i},
\end{gathered}
$$

$b_{i-1}^{2} a_{i} d_{i-1}^{2} \leadsto b_{i-1} a_{i} d_{i-1} d_{i}^{2} b_{i-1} b_{i} d_{i-1} b_{i}, \quad b_{i-1}^{2} b_{i} d_{i-1}^{2} \leadsto b_{i-1} b_{i} d_{i-1} d_{i}^{2} b_{i-1} b_{i} d_{i-1} b_{i}$, $b_{i-1}^{2} c_{i} d_{i-1}^{2} \leadsto d_{i} b_{i-1} d_{i} d_{i-1} b_{i}^{2} b_{i-1} c_{i} d_{i-1}, \quad b_{i-1}^{2} d_{i} d_{i-1}^{2} \leadsto d_{i} b_{i-1} d_{i} d_{i-1} b_{i}^{2} b_{i-1} d_{i} d_{i-1}$.

Как мы показали, все указанные преобразования разлагаются в композицию локальных преобразований (1)-(3). Заменяя буквы $\left\{a_{i}, b_{i}, c_{i}, d_{i}\right\}$ на $\bullet, b_{i-1}$ на ( и $d_{i-1}$ на $)$, получаем следуюшую интерпретацию указанных выше преобразований:

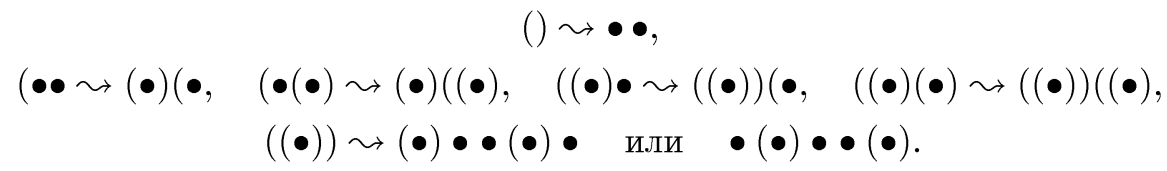

Легко видеть, что процесс остановится через конечное число шагов.

Запишем теперь элементарные движения из предложения 2 алгебраически с помощью алфавита $\mathbb{A}$.

Элементарные движения типа I локальны и превращаются в точности в соотношения (1). Элементарные движения типа II записываются следующим обра3ом:

$$
a_{i-1} b_{i-1} w x \sim w y \sim a_{i+1} d_{i+1} w x,
$$

где $w \in \bar{X}_{i}, x, y \in \mathbb{A}, x^{i}=-1=-y^{i}, x^{i+1}=y^{i+1}, x^{i-1}=y^{i-1}$;

$$
x w d_{i-1} c_{i-1} \sim y w \sim x w b_{i+1} c_{i+1},
$$

где $w \in \bar{X}_{i}, x, y \in \mathbb{A}, x^{i}=1=-y^{i}, x^{i+1}=y^{i+1}, x^{i-1}=y^{i-1}$;

$$
x w d_{i-1} b_{i-1} \sim x w \sim x w b_{i+1} d_{i+1},
$$


где $w \in \bar{X}_{i}, x \in \mathbb{A}, x^{i}=1$.

Докажем (8). Пусть $w \sim w_{1} \ldots w_{k}$ - разложение из леммы 7 . Имеем

$$
\begin{aligned}
a_{i-1} b_{i-1} w x & \sim a_{i-1} b_{i-1} w_{1} w_{2} \ldots w_{k} x \sim w_{1} a_{i-1} b_{i-1} w_{2} \ldots w_{k} x \\
& \sim w_{1} w_{2} a_{i-1} b_{i-1} \ldots w_{k} x \sim \ldots \sim w_{1} w_{2} \ldots w_{k} a_{i-1} b_{i-1} x \\
& \sim w a_{i-1} b_{i-1} x \sim w y \\
a_{i-1} b_{i-1} w x & \sim a_{i+1} d_{i+1} w x .
\end{aligned}
$$

Мы применили (2), (4) и (5). Соотношения (9) и (10) доказываются аналогично.

Чтобы разложить элементарные движения типа III в композицию преобразований (1)-(3), мы воспользуемся следующей леммой.

Лемма 8. Мы имеем

$$
\begin{aligned}
a_{i-1} a_{i+1} b_{i+1} d_{i-1} d_{i+1} b_{i-1} & \sim a_{i+1} a_{i-1}, \\
b_{i-1} a_{i+1} b_{i+1} d_{i-1} d_{i+1} b_{i-1} & \sim a_{i+1} b_{i-1}, \\
a_{i-1} d_{i+1} b_{i+1} d_{i-1} d_{i+1} b_{i-1} & \sim d_{i+1} a_{i-1}, \\
b_{i-1} d_{i+1} b_{i+1} d_{i-1} d_{i+1} b_{i-1} & \sim d_{i+1} b_{i-1}, \\
b_{i+1} d_{i-1} d_{i+1} b_{i-1} c_{i-1} c_{i+1} & \sim c_{i+1} c_{i-1}, \\
b_{i+1} d_{i-1} d_{i+1} b_{i-1} d_{i-1} c_{i+1} & \sim c_{i+1} d_{i-1}, \\
b_{i+1} d_{i-1} d_{i+1} b_{i-1} c_{i-1} b_{i+1} & \sim b_{i+1} c_{i-1}, \\
b_{i+1} d_{i-1} d_{i+1} b_{i-1} d_{i-1} b_{i+1} & \sim b_{i+1} d_{i-1} .
\end{aligned}
$$

ДокАЗАТЕльСтво. Пусть $x_{1} \in\left\{a_{i-1}, b_{i-1}\right\}, x_{2} \in\left\{a_{i+1}, d_{i+1}\right\}$. Из (2) и (4)-(5) мы имеем

$$
\begin{aligned}
x_{1} x_{2} b_{i+1} d_{i-1} d_{i+1} b_{i-1} & \sim x_{1} d_{i-1} x_{2} b_{i+1} d_{i+1} b_{i-1} \\
& \sim x_{1} d_{i-1} x_{2} b_{i-1} \sim x_{2} x_{1} d_{i-1} b_{i-1} \sim x_{2} x_{1} .
\end{aligned}
$$

Соотношения (12) доказываются аналогично.

Элементарные движения типа III имеют вид

$$
x_{1} x_{2} w y_{2} y_{1} \sim x_{2} x_{1} w y_{1} y_{2},
$$

где $w \in \bar{X}_{i}, x_{1} \in\left\{a_{i-1}, b_{i-1}\right\}, x_{2} \in\left\{a_{i+1}, d_{i+1}\right\}, y_{1} \in\left\{c_{i-1}, d_{i-1}\right\}, y_{2} \in\left\{c_{i+1}, b_{i+1}\right\}$. Чтобы доказать соотношение (13), мы опять воспользуемся разложением $w \sim$ $w_{1} \ldots w_{k}, w_{1}, \ldots, w_{k} \in X_{i}$. Применяя лемму 8, получаем

$x_{1} x_{2} w y_{2} y_{1} \sim x_{1} x_{2} w b_{i+1} d_{i-1} d_{i+1} b_{i-1} y_{1} y_{2} \sim x_{1} x_{2} w_{1} \ldots w_{k-1} w_{k} b_{i+1} d_{i-1} d_{i+1} b_{i-1} y_{1} y_{2}$

$\sim x_{1} x_{2} w_{1} \ldots w_{k-1} b_{i+1} d_{i-1} d_{i+1} b_{i-1} w_{k} y_{1} y_{2}$

$\sim x_{1} x_{2} w_{1} \ldots b_{i+1} d_{i-1} d_{i+1} b_{i-1} w_{k-1} w_{k} y_{1} y_{2} \sim \ldots$

$\sim x_{1} x_{2} b_{i+1} d_{i-1} d_{i+1} b_{i-1} w_{1} \ldots w_{k-1} w_{k} y_{1} y_{2}$

$\sim x_{1} x_{2} b_{i+1} d_{i-1} d_{i+1} b_{i-1} w y_{1} y_{2} \sim x_{2} x_{1} w y_{1} y_{2}$. 


\section{ЛитеРАТУРА}

1. Brunn H. Über verknotete Kurven, Mathematiker-Kongresses Zurich 1897, Leipzig, 1898, S. 256-259.

2. Cromwell P. R., Nutt I. J. Embedding knots and links in an open book. II: Bounds on arc index. Math. Proc. Cambridge Philos. Soc., 119, No. 2, 309-319 (1996).

3. Morton H. R., Beltrami E. Arc index and the Kauffman poynomial. Math. Proc. Cambridge Philos. Soc., 123, 41-48 (1998).

4. Дынников И. А. Трехстраничное представление зацеплений. УМН, $\mathbf{5 3}$, вып. 5, 237-238 (1998).

5. Dynnikov I. A. A new way to represent links. One-dimensional formalism and untangling technology. Preprint, Moscow 1998, http://mech.math.msu.su/ dynnikov.

6. Тураев В. Г. Операторные инварианты связок и $R$-матрицы. Изв. АН CCCР, сер. матем., 53, № 5, 1073-1107 (1989).

7. Kauffman L. H. State models and the Jones polynomial. Topology, 26, No. 3, 395-407 (1987).

8. Kauffman L. H. An invariant of regular isotopy. Trans. Amer. Math. Soc., 318, No. 2, 417-471 (1990).

Московский государственный университет, механико-математический факультет

Поступило в редакцию e-mail: dynnikov@mech.math.msu.su 